\title{
Optimization of Linear Dipole Antenna Array for Sidelobe Reduction and Improved Directivity using APSO algorithm
}

\author{
P Victoria Florence ${ }^{1}$, Prof. G.S.N. Raju ${ }^{2}$ \\ ${ }^{1}$ Research Scholar, Dept. of ECE, A U College of Engineering (A), Andhra University Visakhapatnam, India. \\ ${ }^{2}$ Professor, Dept. of ECE, A U College of Engineering (A), Andhra University Visakhapatnam, India.
}

\begin{abstract}
Design of non-uniformly spaced linear array of dipoles is presented. Array synthesis is done by controlling the appropriate excitation amplitudes and element spacing to get the radiation patterns with reduced sidelobe level (SLL) and improved beam width. The objective of this work is to generate synthesized patterns with low sidelobe level and to compensate the coupling effect with a low prefixed dynamic range ratio for non-uniformly spaced array. The excitation amplitude distribution and position of individual array elements are determined. The patterns of dipole elements are compared with those of the isotropic elements. Numerical results show the effectiveness of the algorithm for the design of the linear array.
\end{abstract}

Key words: Pattern synthesis, linear array, low sidelobe level, APSO

\section{Introduction}

Antenna array design with high directivity and low sidelobes are the essential requirements in producing a desired radiation pattern. The process of creating a desired far-field radiation pattern by combining multiple elements in an array is called Antenna pattern synthesis. The pattern synthesis of an antenna array is related to the excitation and geometrical configuration of its elements. The geometry of the antenna array deals with the spacing between the elements and the excitation involves both the amplitude and the phase. The geometry of the array combinations is optimized to achieve the desired performance. Many techniques have been evolved on the design of antenna array synthesis in order to produce patterns with several requirements [13].c

Modern array antenna synthesis methods have achieved considerable success in producing a wide range of versatile and high quality radiation patterns. Many approaches have been attempted to reduce the SLL of the linear array by amplitude tapering, space tapering, phase tapering or by optimizing both amplitudes and spacing [4-6]. But a phase taper has a very limited success in reducing the SLLs. Thinning of an array is another technique for achieving low SLL [7]. Minimization of SLL is the main quality requirement to be satisfied in solving pattern synthesis problem as low sidelobe antenna arrays can reject interference outside the main beam.

Aperiodic arrays are antenna arrays with unequally spaced elements. Unequally spaced arrays have some interesting characteristics which an equally spaced array cannot offer [8]. For the last few years, study of unequally or non-uniformly spaced arrays has attracted the attention of many researchers. Low SLLs can be easily achieved by applying non-uniform excitation on the array elements. Different theoretical approaches on unequally spaced arrays were proposed to achieve the required pattern [9]. Skolnik [10] used a systematic procedure called dynamic programming to design an unequally spaced array. King [11], proposed a method for the reduction of sidelobes and grating lobes using unequally spaced arrays. A pertubational procedure to reduce the SLLs of uniformly excited and non-uniformly spaced linear arrays were developed by Harrington [12].

Synthesis of an antenna array is a highly non-linear and most important electromagnetic optimization problem. Analytical methods have become inefficient to deal with such complex problems involving several parameters. Therefore, evolutionary optimization tools such as Genetic Algorithm [13], Differential Evolution [14], Simulated Annealing [15] etc., are used to synthesize an array for different applications. Particle Swarm Optimization (PSO) algorithms are successfully applied in electromagnetic radiation problems [16-18].

Antenna array generally consists of identical elements. Various types of practical radiating elements are used in arrays to achieve the desired directional characteristics. The commonly used practical radiating elements are dipoles, monopoles, waveguides, horns and slots etc., depending on the application. The configuration considered is a linear array structure with basic antenna elements as isotropic radiators and dipole radiators. The resultant radiation patterns depend on the array factor and element pattern. In the case of isotropic radiators the element pattern is taken as unity and for the case of dipole radiators the element pattern of dipole is taken into account.

A new nature inspired meta-heuristic optimization algorithm, Accelerated Particle Swarm optimization (APSO) [19], a variant of PSO is used for the synthesis of linear array to obtain the lowest possible SLL. The Accelerated PSO algorithm is based on the movement and intelligence of swarms. In the present work, the synthesis of linear arrays with non-uniform excitation amplitudes and non-uniform spacing between the array 
elements are determined using APSO algorithm. The radiation patterns of the dipole elements are compared to those of the isotropic elements. Optimization is carried out to compute the excitation and geometry of individual array elements that will generate low SLLs with prefixed dynamic range ratio (DRR). The main concern of the paper is to find the best arrangement of linear array of dipoles which can give a relative SLL as low as possible with coupling effect taken into consideration. Mutual coupling is compensated by prefixing DRR at lower value. The results obtained have shown the applicability of the APSO to antenna design problems and the patterns are synthesized for different number of elements.

The rest of this paper is organized as follows. In Section II, The synthesis of linear antenna array geometry, Formulation of the array factor and Fitness function are explained. Section III, deals with the concepts of Accelerated Particle Swarm Optimization. The synthesized patterns with reduced sidelobe level for different array elements are presented in Section IV. Finally, the conclusions are drawn in Section V.

\section{Problem Formulation}

An array of $2 \mathrm{~N}$ number of identical half-wavelength dipoles spaced a distance $\mathrm{d}_{\mathrm{n}, \mathrm{n}-1}$ apart along the $\mathrm{z}$ axis as shown in Fig. 1 is considered

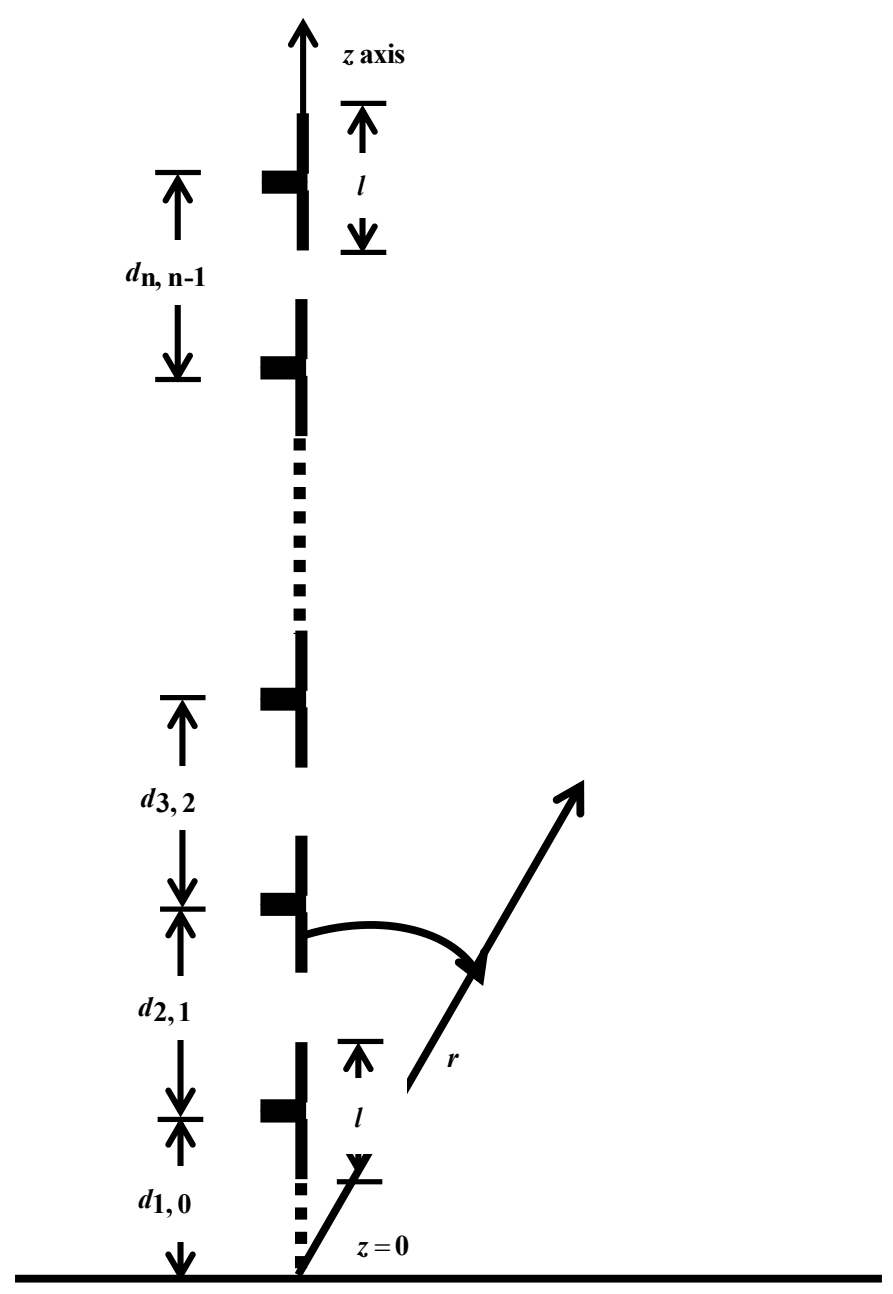

Fig 1. Linear array of dipoles along z-axis 
Assuming sinusoidal current distribution of dipole antenna directed along the $\mathrm{z}$ - axis, the element pattern is calculated using Equ. (1).Excitation and geometry are assumed symmetric with respect to the centre of the array. The far field $\operatorname{AF}(\theta)$ pattern in the horizontal xy plane considering the element pattern, $F(\theta)$ is given by

$$
\begin{aligned}
& \mathrm{F}(\theta)=\frac{\cos (0.5 \pi \cos \theta)}{\sin \theta} \\
& \mathrm{AF}(\theta)=\sum_{n=1}^{N} 2 \mathrm{I}_{\mathrm{n}} \cos \left(\mathrm{kd}_{\mathrm{n}} \cos \theta\right) \times \mathrm{F}(\theta)
\end{aligned}
$$

Normalized radiation pattern in $\mathrm{dB}$ can be expressed as follows.

$$
\mathrm{AF}_{\mathrm{n}}(\theta)=20 \log _{10}\left[\frac{|\mathrm{AF}(\theta)|}{|\mathrm{AF}(\theta)|_{\max }}\right]
$$

Here, $n$ is the element number, $k=2 \pi / \lambda$ is the wave number, $\lambda$ is the wavelength, $d_{n}$ is the distance of centre of the $\mathrm{n}^{\text {th }}$ element from origin, $\mathrm{N}$ is the total number of elements, $\theta$ is the polar angle of the far field measured from broad side.

The radiation pattern produced by the array is required to satisfy the condition of low SLL and optimum dynamic range ratio (DRR). DRR is the ratio of maximum value of excitation amplitude to the minimum value. To optimize the arrays according to the above two conditions, the fitness function is expressed as

$$
\text { Fitness }=\mathrm{a} \times \text { Minimize }\left\{\operatorname{Max}\left(\mathrm{AF}_{\mathrm{n}}(\theta)\right)\right\}+\mathrm{b} \times\left(\mathrm{DRR}-\mathrm{DRR}_{\mathrm{des}}\right)^{2}
$$

Here $\mathrm{a}$ and $\mathrm{b}$ are the weight factors, DRR, $\mathrm{DRR}_{\text {des }}$ are obtained and desired values of the corresponding terms. The Accelerated PSO algorithm attempts to minimize the fitness function to meet the desired pattern specification.

\section{Accelerated Particle Swarm Optmization}

PSO is an evolutionary algorithm based on the behavior of swarm of bees and flock of birds generally seen in nature [20]. PSO was developed by Kennedy and Eberhart in the year 1995. PSO algorithm is based on the principle that each solution can be represented as a particle in a swarm. It depends on the movement and intelligence of swarms which consists of agents called particles. These particles are allowed to fly within the solution space randomly without any prior knowledge. Each agent has a position and velocity vector and each position coordinate represents a parameter value. The algorithm evaluates the fitness function, the position with the best value in the entire search is called the global best, gbest and the individual best value is called as personal best, pbest. Initially each agent is initialized with random position and velocity. For each iteration, the velocity and position of the particles are updated by using the following equations respectively.

$$
\begin{aligned}
& \mathrm{V}_{\mathrm{n}}(\mathrm{t}+1)=\mathrm{w} \cdot \mathrm{V}_{\mathrm{n}}(\mathrm{t})+\mathrm{c}_{1} \cdot \mathrm{r}_{1}\left(\text { pbest }_{\mathrm{n}}-\mathrm{X}_{\mathrm{n}}(\mathrm{t})\right)+\mathrm{c}_{2} \cdot \mathrm{r}_{2}\left(\text { gbest }-\mathrm{X}_{\mathrm{n}}(\mathrm{t})\right) \\
& \mathrm{X}_{\mathrm{n}}(\mathrm{t}+1)=\mathrm{X}_{\mathrm{n}}(\mathrm{t})+\mathrm{V}_{\mathrm{n}}(\mathrm{t}+1)
\end{aligned}
$$

Here, $w$ is the inertia coefficient of the particle which plays an important role in PSO was introduced by Eberhart and Shi [21]. $V_{n}(t+1)$ is the current particle velocity, $V_{n}(t)$ is the previous particle's velocity, $X_{n}$ $(t+1)$ is the current particle's position, $X_{n}(t)$ is the previous particle's position. $r_{1}$ and $r_{2}$ are uniformly distributed random numbers in the range [0.1]. $c_{1}$ and $c_{2}$ are the acceleration constants which control the relative effect of the pbest and gbest particles. pbest is the current personal best value, gbest is the global best value.

A new evolutionary algorithm, variant of PSO which extends the standard PSO algorithm used in the design of linear antenna array is Accelerated Particle Swarm Optimization. APSO was introduced by Xin She Yang in the year 2008. The standard PSO uses both pbest and gbest value, this version of APSO uses only gbest as it could accelerate the convergence of the algorithm faster. The position and velocity vectors of the particles are initialized randomly and are updated with time using Equs. (6-7). These two equations are combined into a single equation which increase the convergence even further is given by

$$
X_{n}(t+1)=(1-\beta) X_{n}(t)+\beta \cdot \text { gbest }+\alpha \cdot c_{n}
$$


The typical values of APSO are $\alpha=0.1$ to 0.4 and $\beta=0.1$ to 0.7 . Here $\alpha$ is taken as 0.2 and $\beta$ as 0.5 . The advantage of using this algorithm is to reduce the randomness as the numbers of iterations proceed. This can be done by using a monotonically decreasing function given by

$\alpha=\gamma^{\mathrm{t}} \quad(0<\gamma<1)$

Here $\gamma$ is the control parameter which is taken as 0.97 and $t$ is the number of iterations or time steps where $t \in\left[0, t_{\max }\right]$ and $t_{\max }$ is the maximum value of the iterations. The performance of APSO algorithm depends on the control parameters. The simulation parameters are carefully chosen. Table 1.1 shows the control parameters for APSO are set as shown below.

Table 1: APSO control parameters

\begin{tabular}{|c|c|}
\hline Parameter & Value \\
\hline Swarm Size & 20 \\
$\alpha$ & 0.20 \\
$\beta$ & 0.50 \\
$\gamma$ & 0.97 \\
$\mathrm{t}_{\max }$ & 100 \\
\hline
\end{tabular}

\section{Results}

In the present work, APSO algorithm is applied for the synthesis of linear arrays. An array of 10, 12, 20 and 24 elements are considered. The excitation current amplitude and inter-element spacing is varied in the range of 0 to 1 and 0.4 to 0.8 wavelengths respectively. All the excitation phases are kept at 0 degree. Desired DRR value of amplitude distribution is prefixed at 7.0. For an array of 10 elements only 5 amplitudes and 4 inter-element distances are to be optimized due to symmetry. The first element is placed at a prefixed distance of 0.2 from the origin. For $\mathrm{N}=10$, the lowest sidelobe level obtained for dipole radiators is about $-36.41 \mathrm{~dB}$. The normalized excitation amplitudes and optimum element positions are shown in Tables 2-5. Figs. (2-9) depicts the optimum excitation amplitudes, positions and corresponding radiation patterns of dipole and isotropic radiators for 10, 12, 20 and 24 elements. Table 6 shows the obtained sidelobe level, null to null beam width, directivity and drr values for the same number of array elements.

Table 2: Excitation amplitudes and spacing for an array of $\mathrm{N}=10$ elements

\begin{tabular}{|c|c|c|}
\hline Element Number & Position $(\lambda)$ & Amplitude \\
\hline $1 \& 1^{\prime}$ & 0.2000 & 0.7778 \\
$2 \& 2^{\prime}$ & 0.6648 & 1.0000 \\
$3 \& 3^{\prime}$ & 1.3085 & 0.9458 \\
$4 \& 4^{\prime}$ & 2.0299 & 0.5532 \\
$5 \& 5^{\prime}$ & 2.7612 & 0.1981 \\
\hline
\end{tabular}

Table 3: Excitation amplitudes and spacing for an array of $\mathrm{N}=12$ elements

\begin{tabular}{|c|c|c|}
\hline Element Number & Position $(\lambda)$ & Amplitude \\
\hline $1 \& 1^{\prime}$ & 0.2000 & 0.7595 \\
$2 \& 2^{\prime}$ & 0.8400 & 1.0000 \\
$3 \& 3^{\prime}$ & 1.5498 & 0.6609 \\
$4 \& 4^{\prime}$ & 1.9741 & 0.2630 \\
$5 \& 5^{\prime}$ & 2.5100 & 0.3645 \\
$6 \& 6^{\prime}$ & 3.2752 & 0.1569 \\
\hline
\end{tabular}


Table 4: Excitation amplitudes and spacing for an array of $\mathrm{N}=20$ elements

\begin{tabular}{|c|c|c|}
\hline Element Number & Position $(\lambda)$ & Amplitude \\
\hline $1 \& 1^{\prime}$ & 0.2000 & 0.9243 \\
$2 \& 2^{\prime}$ & 0.6181 & 0.9695 \\
$3 \& 3^{\prime}$ & 1.0463 & 0.9072 \\
$4 \& 4^{\prime}$ & 1.4912 & 1.0000 \\
$5 \& 5^{\prime}$ & 2.0366 & 0.9936 \\
$6 \& 6^{\prime}$ & 2.6237 & 0.8893 \\
$7 \& 7^{\prime}$ & 3.2581 & 0.7227 \\
$8 \& 8^{\prime}$ & 3.9485 & 0.5599 \\
$9 \& 9^{\prime}$ & 4.7082 & 0.3414 \\
$10 \& 10^{\prime}$ & 5.4302 & 0.1460 \\
\hline
\end{tabular}

Table 5: Excitation amplitudes and spacing for an array of $\mathrm{N}=24$ elements

\begin{tabular}{|c|c|c|}
\hline Element Number & Position $(\lambda)$ & Amplitude \\
\hline $1 \& 1^{\prime}$ & 0.2000 & 0.7289 \\
$2 \& 2^{\prime}$ & 0.8019 & 1.0000 \\
$3 \& 3^{\prime}$ & 1.4081 & 0.7465 \\
$4 \& 4^{\prime}$ & 1.8779 & 0.6287 \\
$5 \& 5^{\prime}$ & 2.4146 & 0.6818 \\
$6 \& 6^{\prime}$ & 2.9824 & 0.5599 \\
$7 \& 7^{\prime}$ & 3.4564 & 0.3818 \\
$8 \& 8^{\prime}$ & 3.9383 & 0.4147 \\
$9 \& 9^{\prime}$ & 4.4762 & 0.2752 \\
$10 \& 10^{\prime}$ & 4.9644 & 0.2650 \\
$11 \& 11^{\prime}$ & 5.6408 & 0.2261 \\
$12 \& 12^{\prime}$ & 6.4397 & 0.1450 \\
\hline
\end{tabular}

Table 6: Obtained Sidelobe level, null to null beam width, directivity and drr values

\begin{tabular}{|c|c|c|c|c|}
\hline $\mathbf{N}$ & SLL (dB) & FNBW(deg) & Directivity & DRR \\
\hline 10 & -36.41 & 33.05 & 4.24 & 5.04 \\
12 & -37.41 & 29.96 & 4.58 & 6.37 \\
20 & -38.89 & 18.39 & 8.72 & 6.89 \\
\hline
\end{tabular}



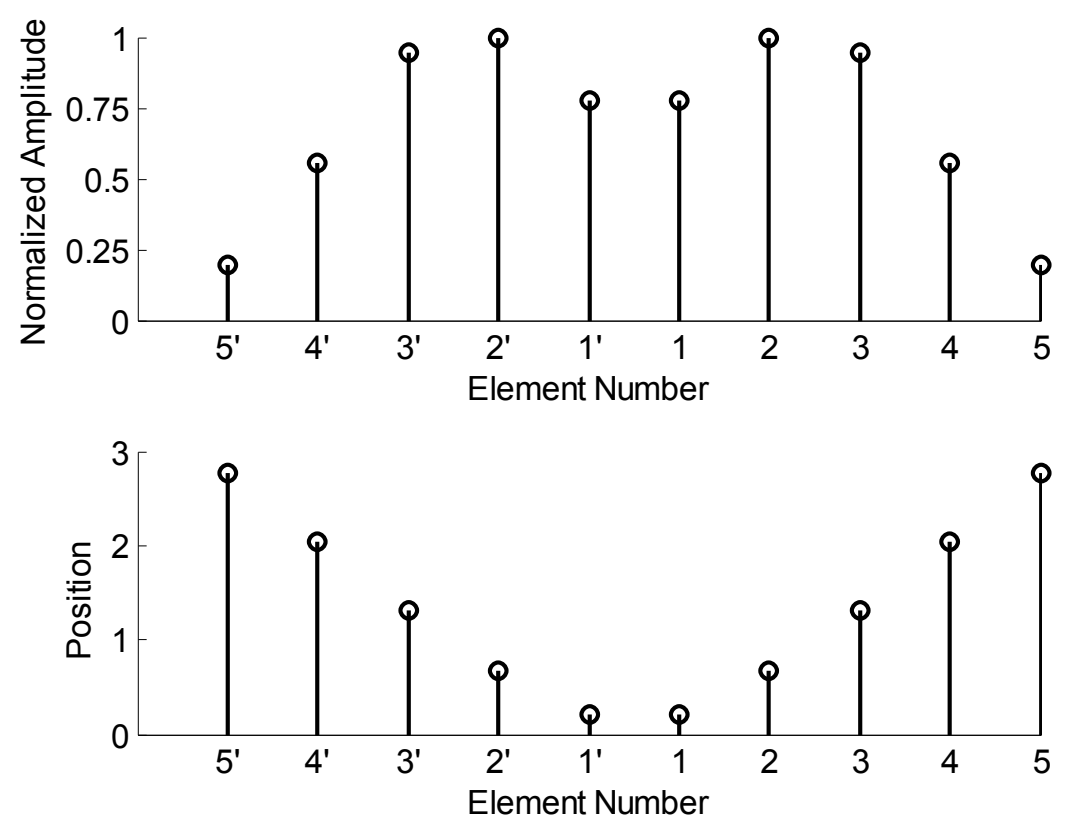

Fig 2.Normalized amplitudes and element positions obtained using APSO for $\mathrm{N}=10$

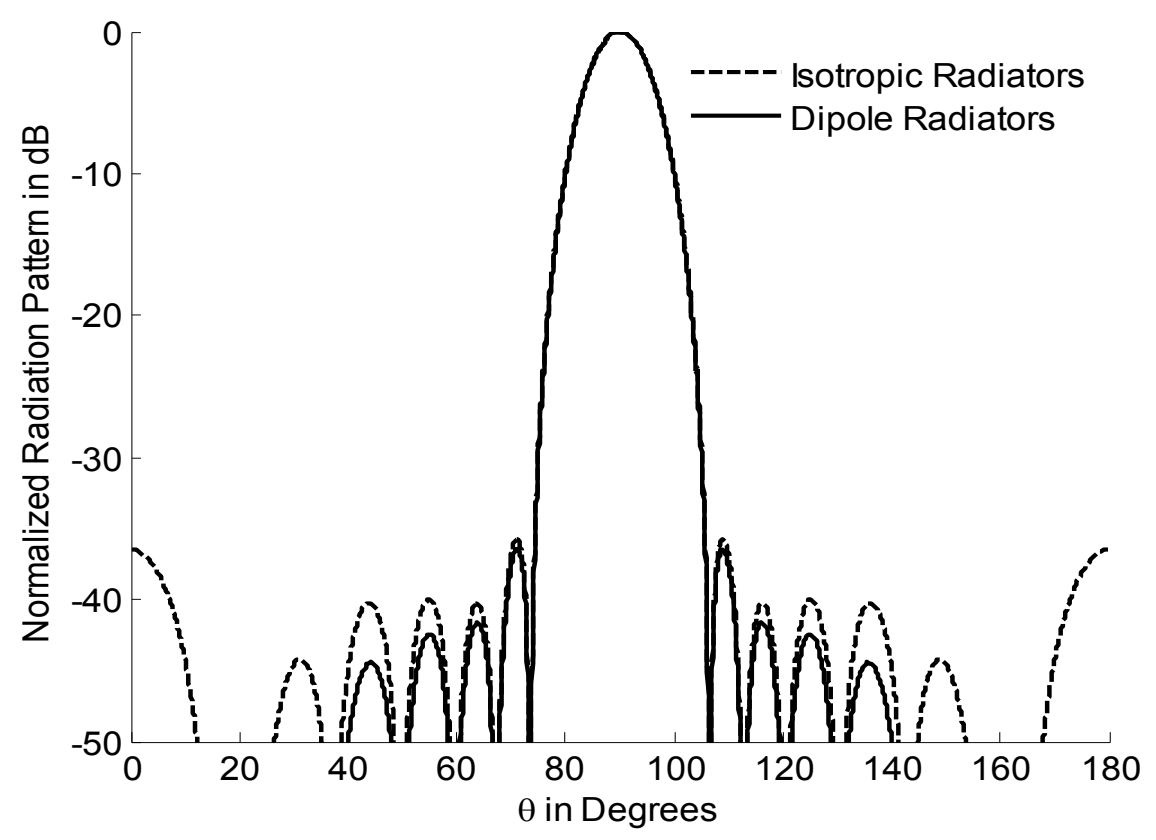

Fig 3. Radiation patterns of isotropic and dipole radiators for an array of 10 elements 

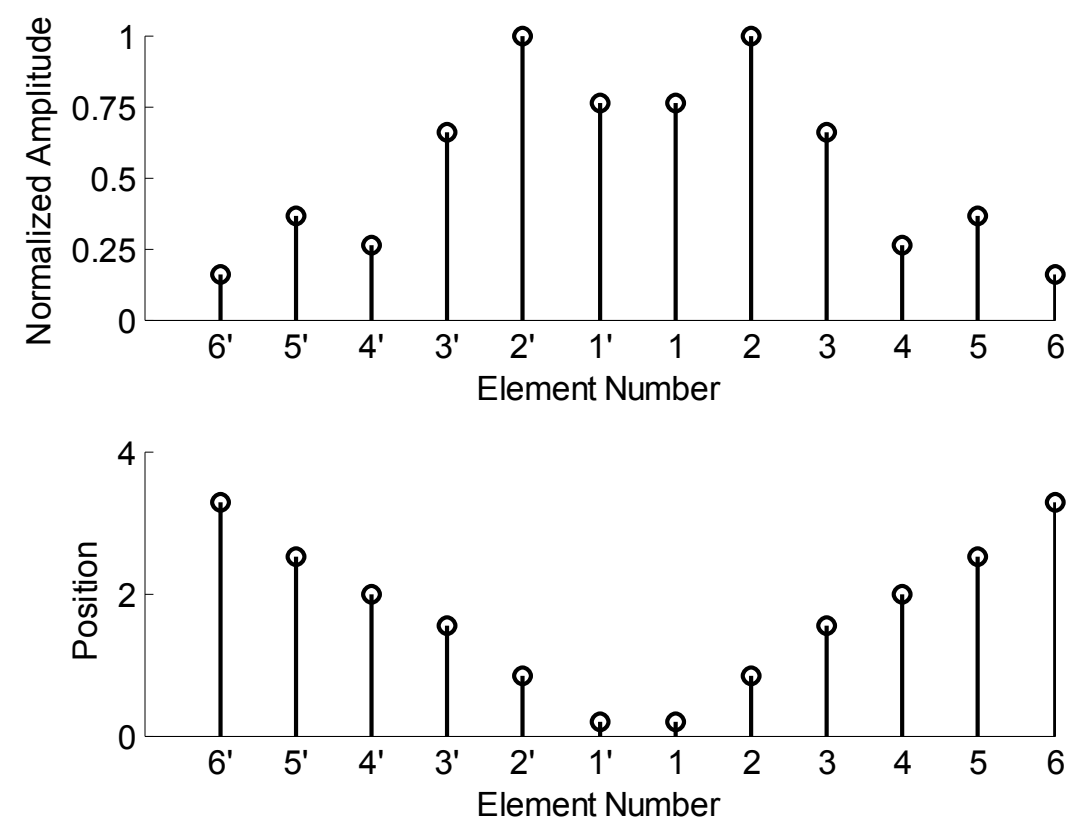

Fig 4.Normalized amplitudes and element positions obtained using APSO for $\mathrm{N}=12$

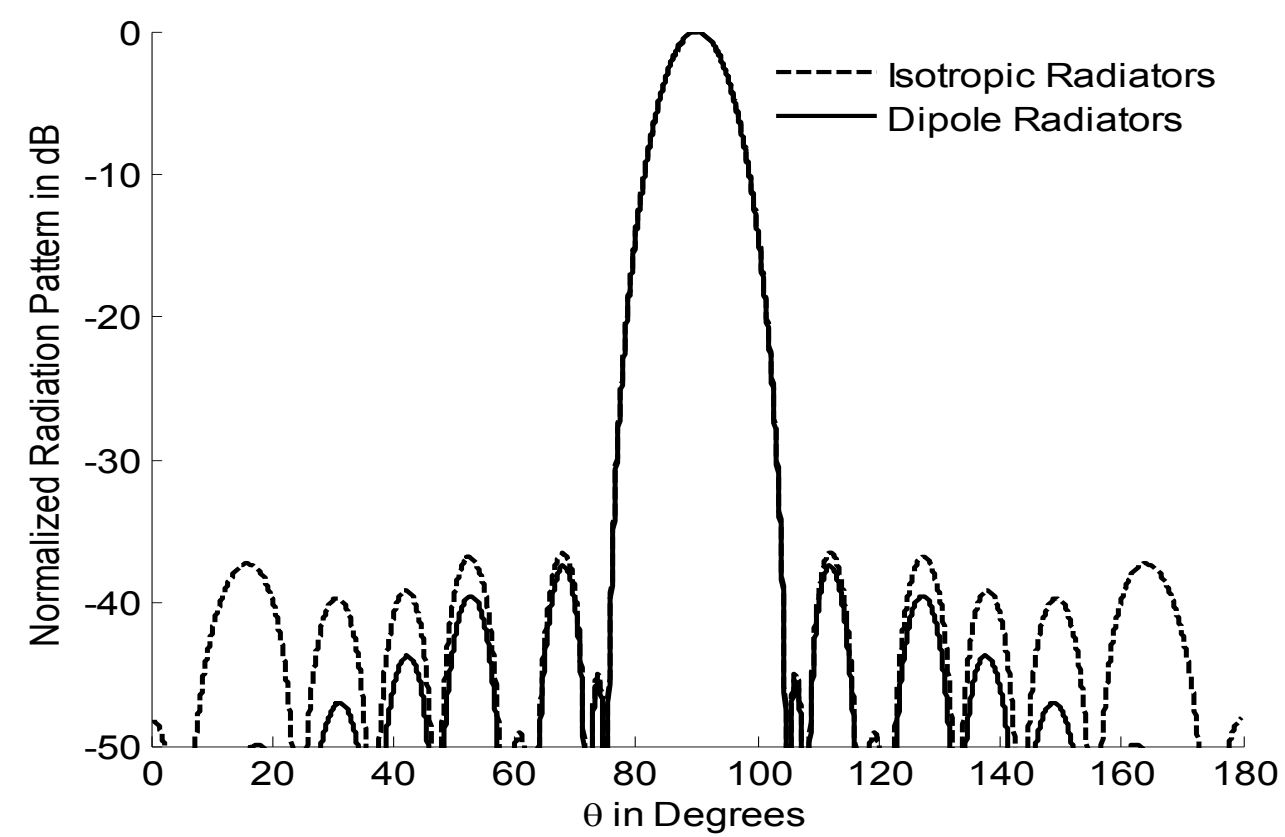

Fig 5. Radiation patterns of isotropic and dipole radiators for an array of 12 elements 

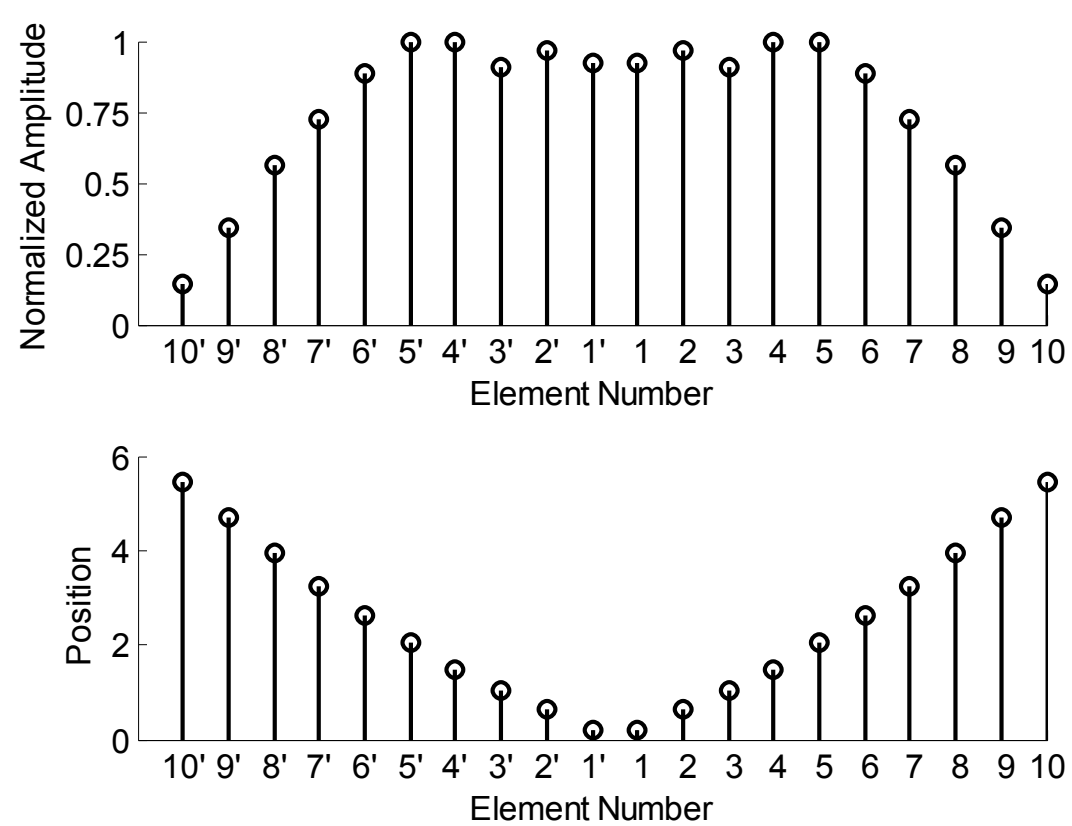

Fig 6.Normalized amplitudes and element positions obtained using APSO for $\mathrm{N}=20$

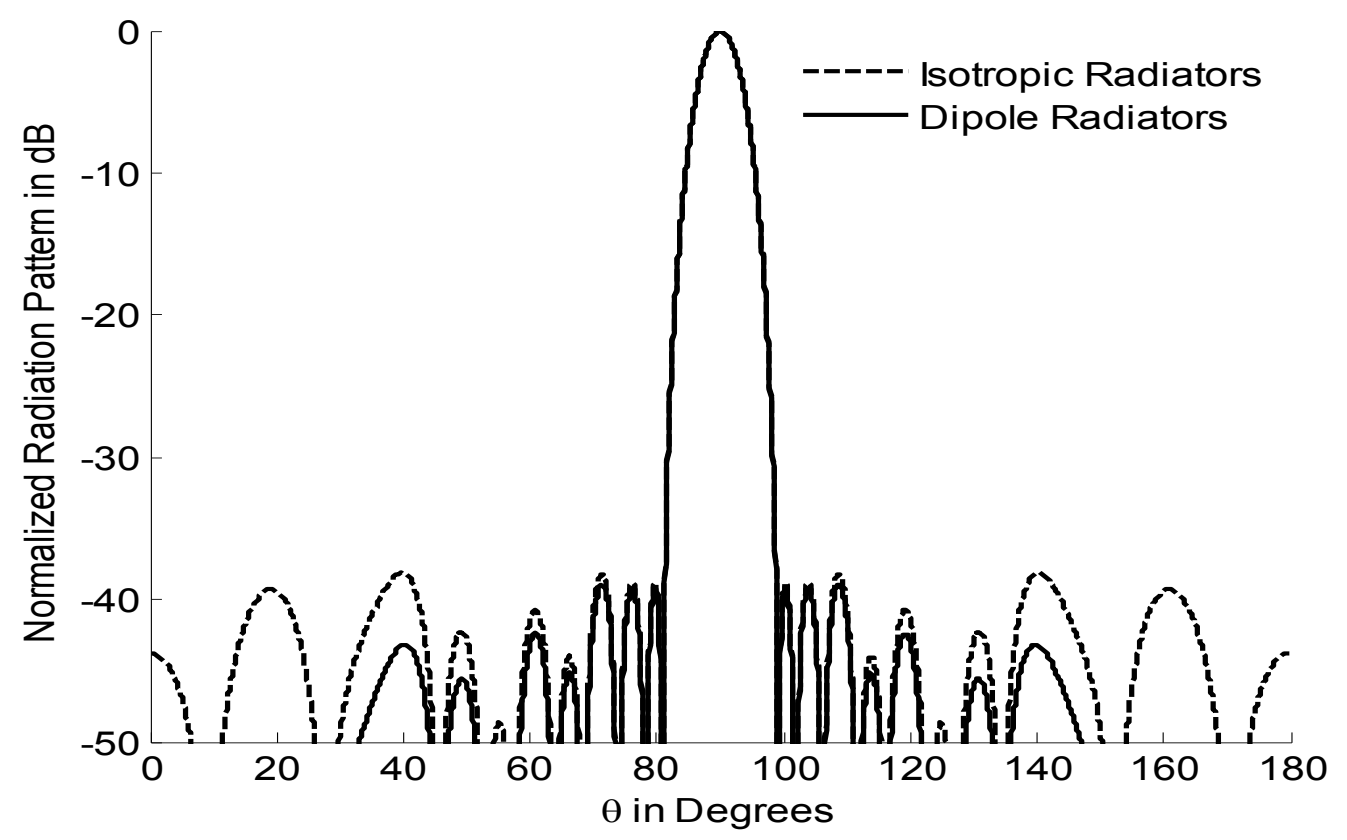

Fig 7. Radiation patterns of isotropic and dipole radiators for an array of 20 elements 

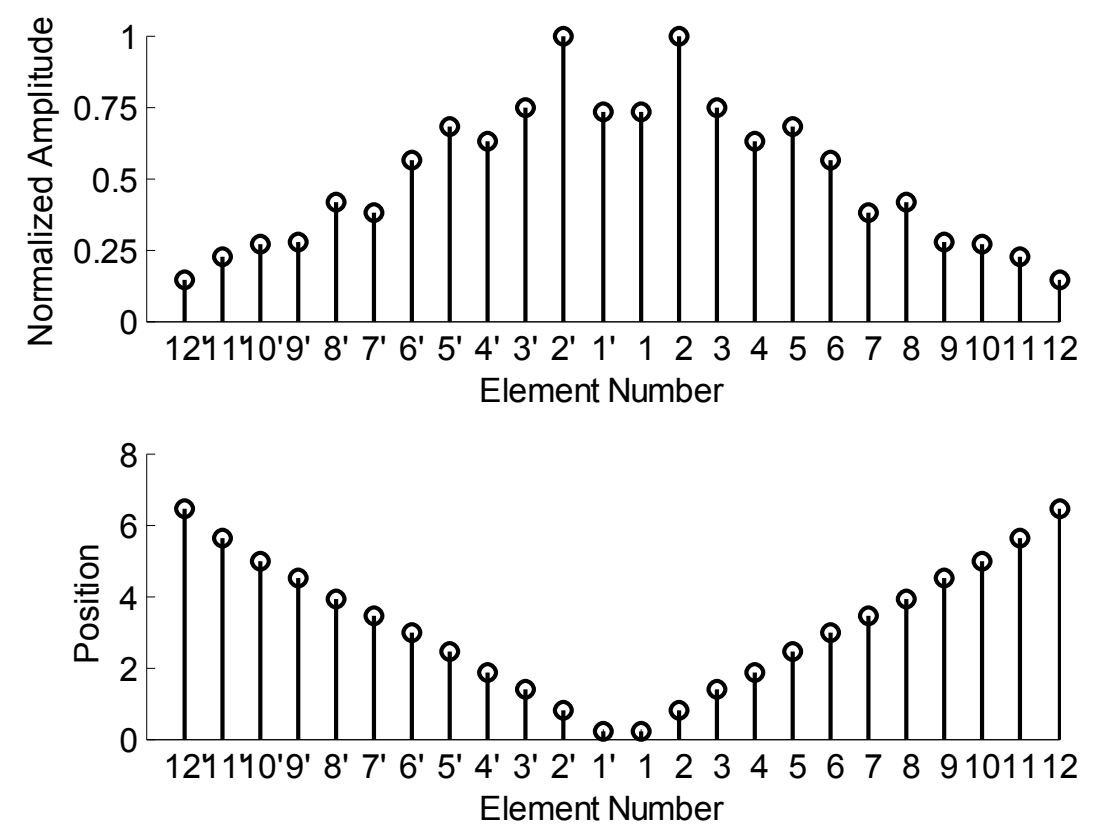

Fig 8.Normalized amplitudes and element positions obtained using APSO for $\mathrm{N}=24$

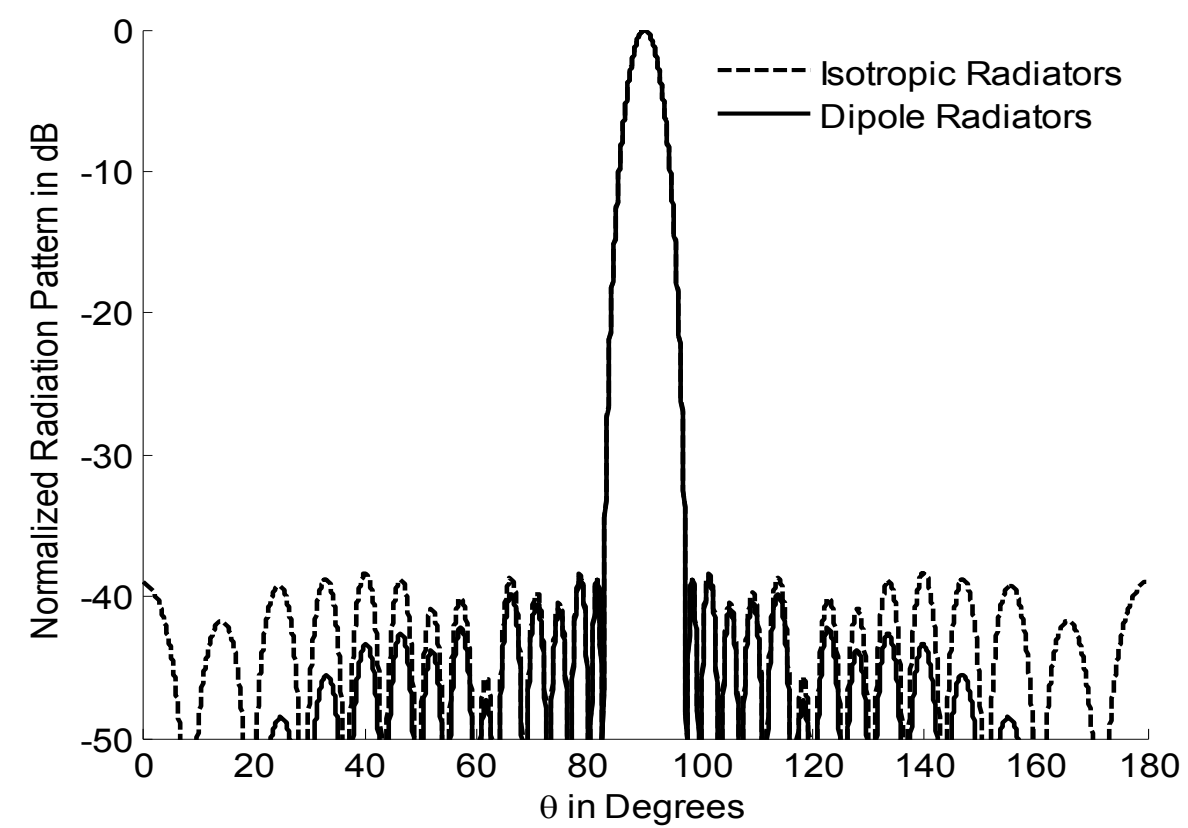

Fig 9. Radiation patterns of isotropic and dipole radiators for an array of 24 elements 


\section{Conclusion}

This paper presents a technique based on Accelerated PSO for the synthesis of non-uniformly spaced linear array. An appropriate excitation amplitude distribution and spacings are determined in order to achieve the low sidelobe level with maximum directivity and fixed DRR. Fixing the DRR of excitation amplitude distribution to a lower value reduces the effect of mutual coupling. The results reveal that the synthesized patterns of the array of dipole radiators possess a considerable relatively low sidelobe level without enhancing the beam width. Thus, APSO is best suited for solving synthesis problems in electromagnetics and this method can be extended to other geometries and constraints.

\section{References}

[1]. J.D.Kraus, Antennas, Mc Graw-Hill International Editions, 1988

[2]. C.A.Balanis, Antenna theory, Analysis and Design, John Wiley and Sons Inc., New York, 1997.

[3]. W.L.Stutzmann, G.A.Thiele, Antenna theory and Design, Wiley, New York, 1981

[4]. R.L.Haupt, "Synthesizing low sidelobe quantized amplitude and phase tapers for linear arrays using genetic algorithms," Proc. Inte. Conf. Electromagnetics in Advanced Applications, Toronto. Italy, September 1995, pp.221 -224.

[5]. P.Lopez, J.A.Rodriquez, F.Ares and E.Mareno, "Low sidelobe level in almost uniformly excited arrays," IEE Electron Letters, November 2000, pp.1991-1993.

[6]. D. G. Kurup, M. Himdi, and A. Rydberg, "Synthesis of uniform amplitude unequally spaced antenna arrays using the differential evolution algorithm," IEEE Transactions on Antennas and Propagation, Vol. 51, September 2003, pp. $2210-2217$.

[7]. R.L.Haupt, "Thinned arrays using Genetic algorithms,” IEEE Transactions on Antennas and Propagation, Vol.42, July 1994, pp. 993-999.

[8]. L.Cen, Z.L.Yu, Wen Cen, "Linear sparse synthesis with minimum number of sensors," IEEE Transactions on Antennas and Propagation, Vol.58, March 2010, pp. 720-726.

[9]. H.Unz, 1960. "Linear arrays with arbitrarily distributed elements," IEEE Transactions on Antennas and Propagation, Vol. 8, March 1960 , pp. 222-223.

[10]. M. I. Skolnik, G. Nemhauser, and J. W. Sherman, 1964. "Dynamic programming applied to unequally spaced arrays," IEEE Transactions on Antennas and Propagation, Vol.12, January 1964, pp. 35-43.

[11]. D.D.King, R.F.Packard and R.K.Thomas, "Unequally spaced broad-band antenna arrays," IRE Transactions on Antennas and propagation, Vol.8, July 1960, pp.380-385.

[12]. R.Harrington, 1961." Sidelobe reduction by nonuniform element spacing," IRE Transactions on Antennas and propagation, Vol.9, March 1961, pp.187-192.

[13]. K.-K. Yan and Y. Lu, "Sidelobe reduction in array-pattern synthesis using genetic algorithm", IEEE Transactions on Antennas and Propagation, Vol. 45, July 1997, pp.1117 -1122.

[14]. C.Lin, A.Qing and Q.Feng, "Synthesis of unequally spaced antenna arrays by using Differential Evolution,” IEEE Trans. Antennas and Propagation, Vol. 58, August 2010, pp.2553 -2561.

[15]. Murino, V.A.Trucco and C.S.Regazzoni, "Synthesis of unequally spaced arrays by simulated annealing", IEEE Trans. On Signal Processing, Vol.44, January 1996, pp.119-122.

[16]. D.Gies and Y.Rahmat Samii, "Particle swarm optimization for reconfigurable phase differentiated array design," Microwave Optical Technology Letters, Vol.38, August 2003, pp.168-175.

[17]. M.M.Khodier, C.G.Christodoulou, "Linear array geometry synthesis with minimum sidelobe level and null control using Particle swarm optimization,” IEEE Transactions on Antennas and Propagation, Vol. 53, August 2005, pp.2674 -2678.

[18]. J.Nanbo and Y.Rahmat Samii, “Advances in particle swarm optimization for antenna designs: Real-number, binary, single-objective and multi-objective implementations," IEEE Transactions on Antennas and Propagation, Vol. 55, March 2007, pp.556 -567.

[19]. X.S.Yang, Nature-Inspired Metaheuristics Algorithms, Luniver Press, 2008.

[20]. Y.Shi, R.C.Eberhart, "Emiprical study of particle swarm optmization," Evolutionary computation, proceedings of the 1999 congress, Vol.3, July 1999.

[21]. J.Kennedy and R.C.Eberhart, "Particle Swarm Optimization", Proceedings of the IEEE International conference on Neural Networks, Vol.4, pp.1942-1948, December 1995.

\section{Author's Information}

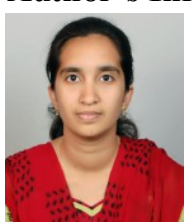

P Victoria Florence received the Bachelor of Technology in Electronics and Communication Engineering in the year of 2007 from JNTU Hyderabad and the Master of Technology in Radar and Microwave Engineering in 2009 from Andhra University College of Engineering (A). Currently, she is working towards her $\mathrm{PhD}$ degree in the department of Electronics and Communication Engineering, Andhra University College of Engineering (A). Her Research interests include Array Antennas, EMI/EMC and Soft Computing. She is a life member of SEMCE (India).

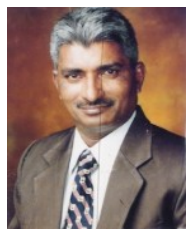

Dr. G.S.N. Raju received his B.E., M.E. with distinction and first rank from Andhra University and Ph.D. from IIT, Kharagpur. At present, he is the Vice - Chancellor of Andhra University and a Senior Professor in Electronics and Communication Engineering. He is in teaching and research for the last 30 years in Andhra University. He guided 28 Ph.D.s in the fields of Antennas, Electromagnetics, EMI/EMC and Microwave, Radar Communications, Electronic circuits. Published about 304 technical papers in National/ International Journals/ Conference Journals and transactions. He is the recipient of The State Best Teacher Award' from the Government of Andhra Pradesh in 1999, 'The Best Researcher Award' in 1994, 'Prof. Aiya Memorial National IETE Award' for his best Research guidance in 2008 and Dr. Sarvepalli Radhakrishnan Award for the Best Academician of the year 
2007, He was a visiting Professor in the University of Paderborn and also in the University Karlsruhe, Germany in 1994. He held the positions of Principal, Andhra University College of Engineering (A), Visakhapatnam, Chief Editor of National Journal of Electromagnetic Compatibility. Prof. Raju has published five textbooks Antennas and Wave Propagation, Electromagnetic Field Theory and Transmission Lines, Electronics Devices and Circuits, Microwave Engineering, Radar Engineering and Navigational Aids. Prof. Raju has been the best faculty performer in Andhra University with the performance index of $99.37 \%$. 